\section{MEK inhibitors: a new class of chemotherapeutic agents with ocular toxicity}

KE Duncan ${ }^{1}$, LY Chang $^{2}$ and M Patronas ${ }^{1}$

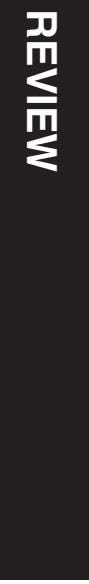

\begin{abstract}
A new class of chemotherapeutic agents, MEK inhibitors, has recently been developed and is proving to be an effective treatment for a number of cancers. A pattern of ocular adverse events has followed these drugs through clinical trials and their association with retinopathy is only just beginning to be recognized. We present two cases of MEK inhibitor-associated retinopathy followed by a review of the current literature on ocular toxicity associated with MEK inhibitors. Patients undergoing treatment with MEK inhibitors appear to have high rates of multifocal serous retinal detachments as well as retinal vein occlusions. We present the first report of cystoid macular edema associated with MEK inhibitor use. The mechanism of these adverse events is still unclear though they seem to be related to oxidative stress and blood retinal barrier breakdown. Management of the ocular toxicity can range from observation to topical treatments or intravitreal injections. Fortunately most ocular adverse events appear to be selflimited and do not require discontinuing the MEK inhibitor. Discontinuation or decreased dosing of MEK inhibitors may be reserved for cases of severe sight-threatening ocular toxicity. Eye (2015) 29, 1003-1012; doi:10.1038/eye.2015.82; published online 5 June 2015
\end{abstract}

\section{Introduction}

Retinal toxicity has been associated with the recent use of a promising class of drugs that has been developed for the treatment of metastatic cancer. These drugs inhibit the mitogenactivated protein kinase/extracellular signalregulated kinase (MAPK/ERK) kinase, also known as the MEK enzyme. Despite significant ocular toxicity associated with these medications, very little information on this topic is present in the ophthalmologic literature.
As MEK inhibitors progress through clinical trials and into the general patient population, eye care professionals should be aware of these medications and their potential ocular toxicity to recognize complications early and preserve vision where possible. We report two cases of as a review of the current literature on these medications and their ocular toxicity.

\section{Case 1}

A 51-year-old female presented for an eye exam prior to starting a clinical trial with a MEK inhibitor for metastatic ovarian cancer. Her vision was $20 / 25$ OU with a normal dilated fundus exam. The patient returned for a repeat exam 2 weeks after initiating MEK 162 at $45 \mathrm{mg}$ PO BID. She had no visual complaints, however, vision was 20/40 OD and 20/25 OS. Retinal exam revealed multifocal creamy yellow deep retinal lesions (Figure 1a). Optical coherence tomography (OCT) revealed thickening and elevation of the retinal pigment epithelium (RPE) at these locations (Figure 2a). Fluorescein angiography (FA) showed early hyperfluorescence and late staining of the lesions in the right eye (Figure 3) and no abnormalities in the left eye. Since the lesions were not vision threatening, it was recommended that she continue the medication at the same dose with close monitoring of the retinal findings. The patient returned in 2 weeks for repeat exam at which time the lesions had decreased in size. Her vision returned to baseline and the lesions had almost completely disappeared at 1-month followup (Figures $1 b$ and $2 b$ ).

CT scan 2 months into therapy revealed that her cancer had a partial response with decrease in the size and number of metastases. At last exam, 6 months after starting the medication, there had been no recurrence of retinal pathology. MEK inhibitor-associated retinal toxicity as well
${ }^{1}$ Department of Ophthalmology and Visual Sciences, University of Maryland School of Medicine, Baltimore, $M D$, USA

${ }^{2}$ University of Maryland School of Medicine, Baltimore, MD, USA

Correspondence: KE Duncan, Department of Ophthalmology and Visual Sciences, University of Maryland School of Medicine, 419 West Redwood Street, Suite 479, Baltimore, MD 21201, USA

Tel: +1 443421 6062;

Fax: +1 4103281178

E-mail: kduncan@umm.edu

Received: 9 November 2014 Accepted in revised form: 14 April 2015

Published online:

5 June 2015 


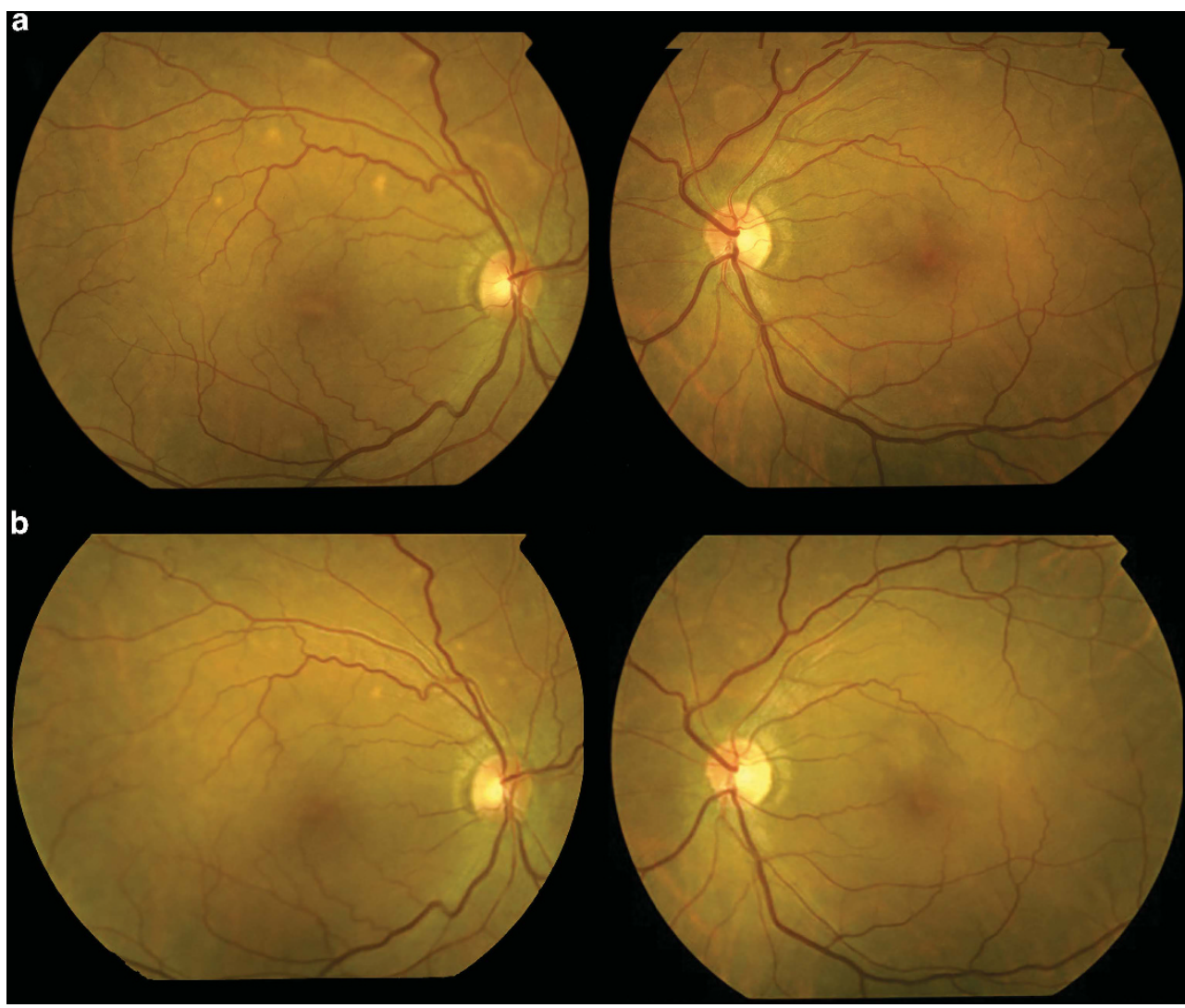

Figure 1 Case 1 fundus photography. (a) Multifocal deep retinal lesions appearing 2 weeks after initiating MEK inhibitor therapy. (b) Improvement in retinal lesions 1 month after initiating MEK inhibitor therapy.

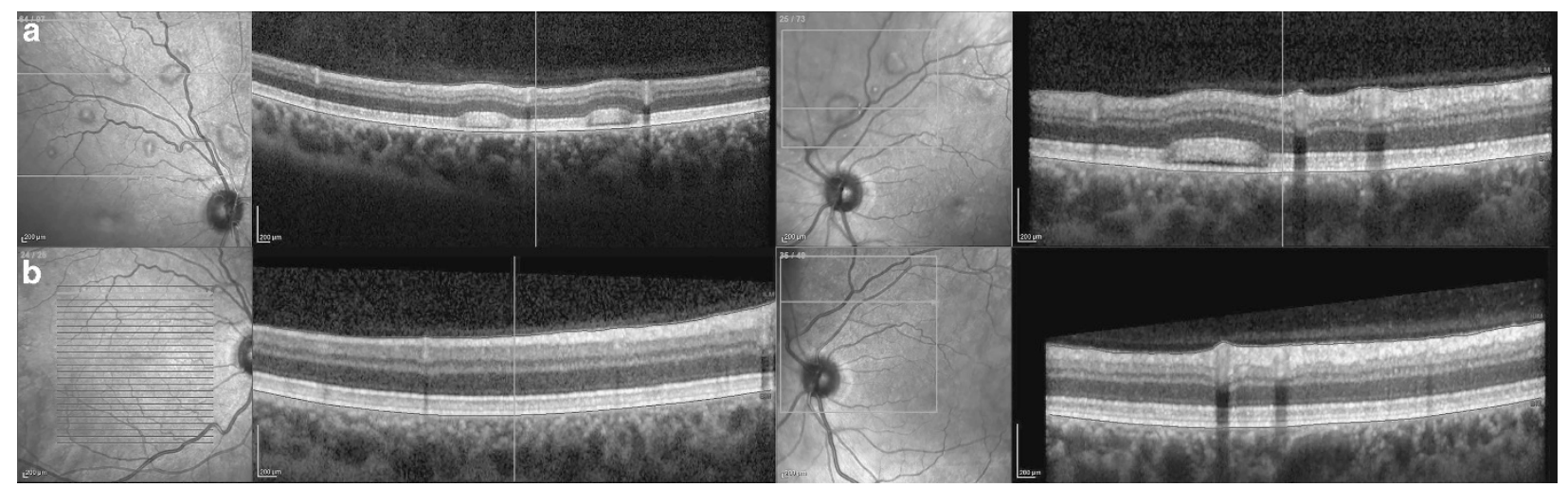

Figure 2 Case 1 optical coherence tomography (OCT). (a) Thickening and elevation of the neurosensory retina and RPE in the area of the retinal lesions noted 2 weeks after initiating MEK inhibitor therapy. (b) Resolution of findings on OCT 1 month after initiating MEK inhibitor therapy.

\section{Case 2}

A 58-year-old male with metastatic melanoma since 2008 presented to the ophthalmology clinic with complaints of blurred vision from the left eye for 3 weeks. He had been started on Trametinib, the only FDA-approved MEK inhibitor, 8 months prior to presentation. Visual acuity was 20/20 OD and 20/60 OS with normal intraocular pressure. Retinal exam and OCT revealed cystoid macular edema (CME) in the left eye (Figure 4a). FA showed late petalloid leakage in the left macula and mild staining of the left optic nerve head (Figure 5). The patient had no history of diabetes, uveitis, macular degeneration, eye surgery, vein occlusions, or any other etiology to explain 
his macular edema. He was started on Pred Forte and Acular QID OS and on follow-up 6 weeks later he showed complete resolution of the CME (Figure $4 b$ ) with return of

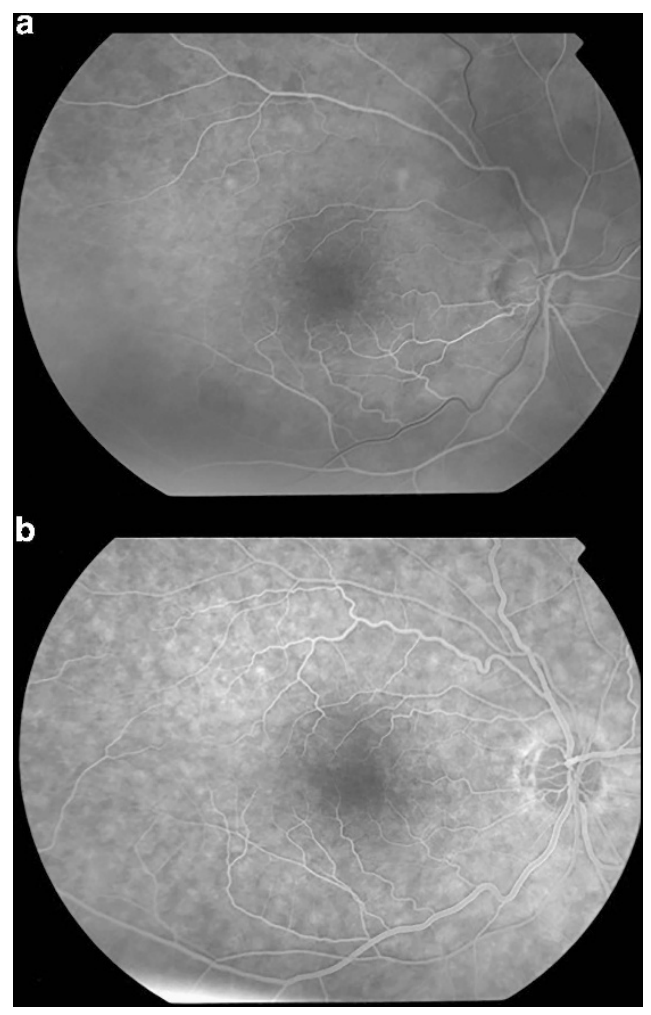

Figure 3 Case 1 fluorescein angiography in the right eye 2 weeks after initiating MEK inhibitor therapy. (a) Hyperfluoresence of retinal lesions was noted in the early phase. (b) Late staining of the retinal lesions was noted in the late phase. visual acuity to $20 / 20$. Following a slow taper of the topical anti-inflammatory drops, the patient noticed slight blurring of the vision 1 week after discontinuation of the treatment. OCT revealed early recurrence and the treatment was re-initiated and the edema subsequently resolved. The patient is being maintained on a daily drop of Pred Forte to prevent recurrence.

\section{Literature review}

This article aims to review the available data on MEK inhibitor ocular toxicity, discuss its pathogenesis, and provide recommendations for management. The mitogenactivated protein kinase (MAPK) pathway is an important signal transduction pathway that controls cellular proliferation. ${ }^{1}$ The pathway includes a series of protein kinases (RAS, RAF, MEK, and ERK), which phosphorylate and activate one another, ultimately resulting in the activation of transcription factors in the cell nucleus that influence gene transcription and subsequently cellular proliferation (Figure 6). Dysregulation of this pathway is commonly found in human cancers. Mutations in the pathway occur most commonly in the RAS and RAF protein kinases. RAS mutations have been isolated in $63 \%$ of pancreatic cancers, $19 \%$ of non-small cell lung cancers, and $36 \%$ of colorectal and thyroid cancers. ${ }^{2}$ RAF mutations have been found in $69 \%$ of papillary thyroid cancers, $59 \%$ of melanomas, $18 \%$ of colorectal cancers and $14 \%$ of liver cancers. ${ }^{3,4}$ As MEK is immediately downstream from RAS and RAF in the signal transduction pathway, it has become a highly attractive chemotherapeutic target.

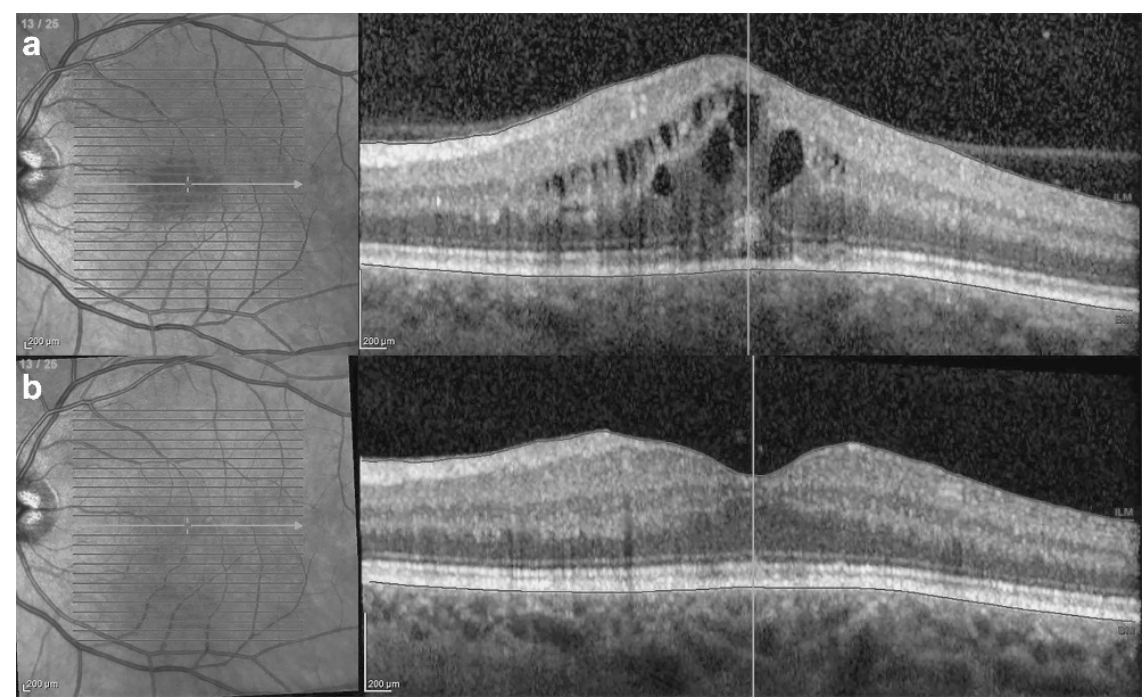

Figure 4 Case 2 optical coherence tomography (OCT) of the left eye. (a) Cystoid macular edema. (b) Resolution of cystoid macular edema after 6 weeks of Pred Forte and Acular. 


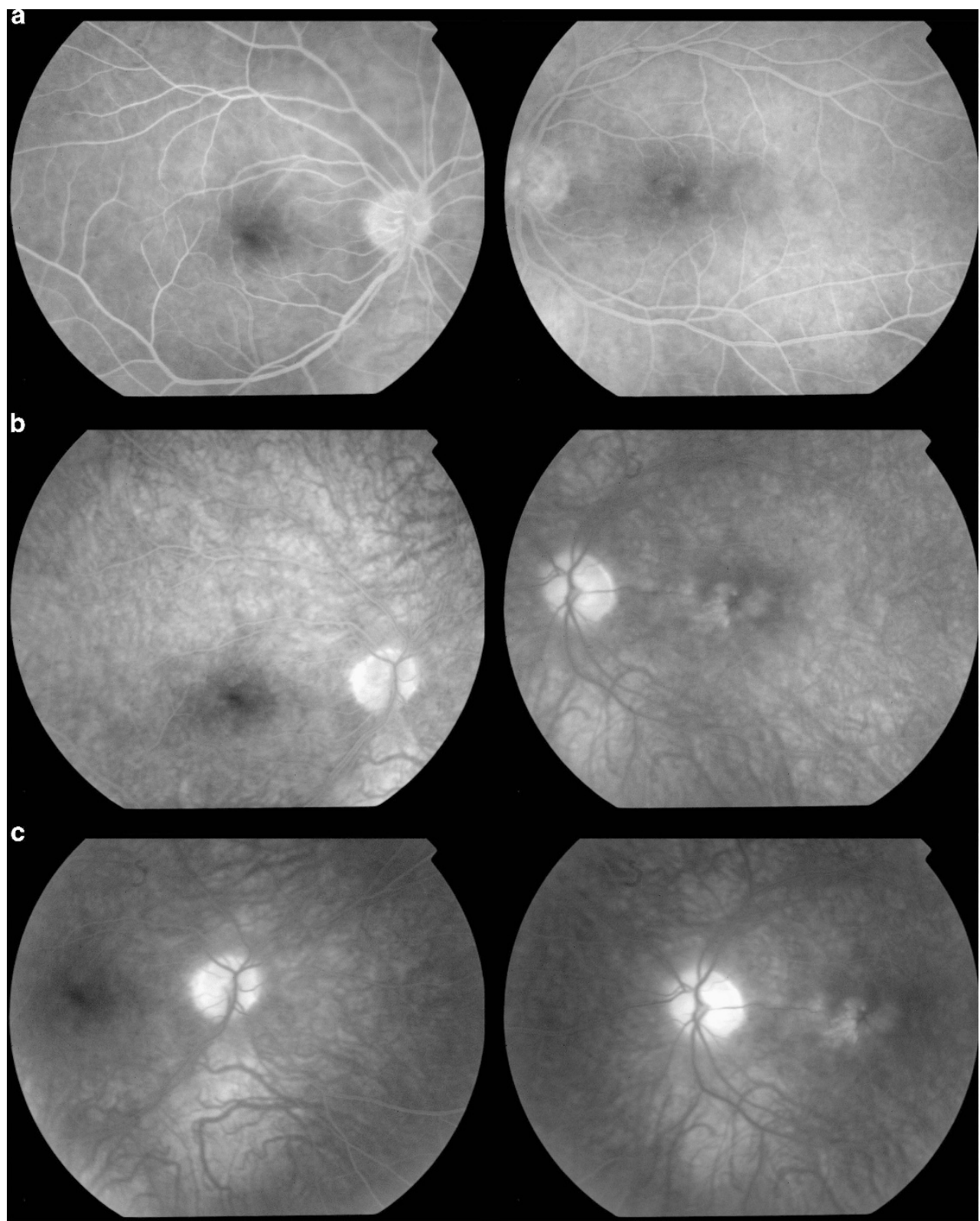

Figure 5 Case 2 fluorescein angiography (FA). Early (a), middle (b), and late (c) phases demonstrating late petalloid leakage of the left macula and staining of the left optic nerve head.

PD098059 was the first MEK inhibitor developed in 1995. Due to poor pharmacokinetics and solubility, its use in vivo was limited. ${ }^{5}$ CI-1040 was the first to enter clinical trials in 2000. 2,6 Since that time, at least 13 different MEK inhibitors have been developed and evaluated in clinical trials. Recent clinical trials have used MEK inhibitors alone and in combination with other chemotherapeutic agents to treat a variety of cancers including melanoma, ovarian cancer, leukemia, lymphoma, thyroid cancer, colorectal cancer, non-small cell lung cancer, biliary cancer, and pancreatic cancer and so on. ${ }^{2}$ Trametinib was the first MEK inhibitor to be approved by the FDA for the treatment of metastatic or unresectable melanoma in May 2013. It remains the only FDA-approved MEK inhibitor available at this time.

As MEK inhibitors progress through clinical trials, numerous drug toxicities have been identified. Similar to other chemotherapeutic agents, the most common adverse events reported have been diarrhea, nausea, rash, and weakness. ${ }^{7-13}$ Unique to MEK inhibitors, however, is the identification of high rates of ocular toxicity emerging in these clinical trials. 


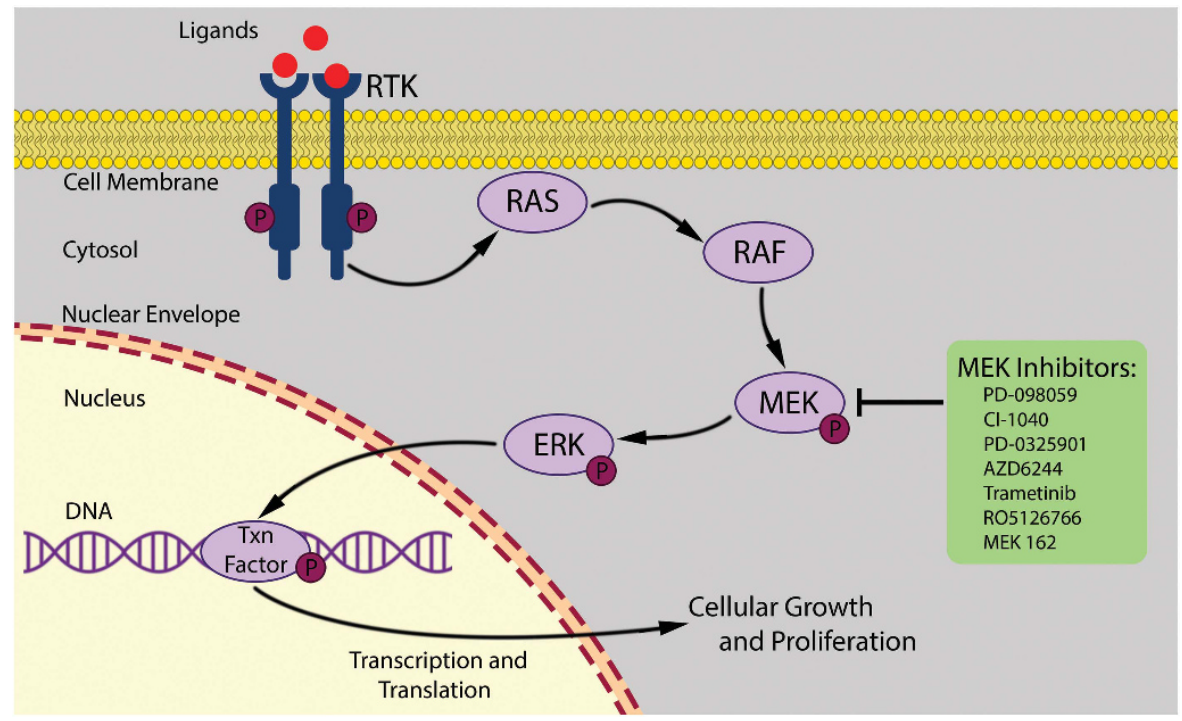

Figure 6 The RAS/RAF/MEK/ERK signal transduction pathway. Extracellular signaling ligands bind to receptor tyrosine kinases in the cell membrane. This results in activation of RAS, which in turn activates RAF. RAF phosphorylates and activates MEK. MEK phosphorylates and activates ERK. Once activated, ERK translocates to the nucleus where it binds to transcription factors to stimulate cell growth and proliferation. Mutations resulting in abnormal activation of this pathway may contribute to many human cancers.

\section{CI-1040}

CI-1040 was the first MEK inhibitor to enter clinical trials in 2000. The phase I trial enrolled 77 patients with locally advanced or metastatic cancer who had failed conventional therapy or for whom there was no established life-prolonging therapy available. The study enrolled patients with 13 different tumor types, with the most frequent being colorectal, non-small cell lung, exocrine pancreatic, melanoma and kidney. About 59.6\% of patients experienced one or more adverse events related to therapy, though $98 \%$ were grade 1 or 2 in severity. There were no reported ocular adverse events. About 66 of these patients were assessed for response to treatment and almost 30\% achieved stabilization of disease lasting a mean of 5.5 months. ${ }^{8}$

Given the success and tolerable side effect profile demonstrated in the phase I study, CI-1040 was further evaluated with a phase II study in which more continuous and increased dosing was used. Sixty-seven patients with breast, colon, non-small cell lung, and pancreatic cancers were enrolled in the study. Visual changes were reported in six $(8.9 \%)$ patients. The authors describe these as 'transient blurring and altered light perception.' One patient had an abnormal retinal exam that subsequently returned to normal after stopping the medication. Vision changes resolved within 1 day, with the exception of one patient whose vision changes persisted for 2 weeks. All patients resumed treatment after visual changes resolved and none of the patients experienced a recurrence of visual symptoms. ${ }^{9}$
In the phase II clinical trial for CI-1040, no partial or complete responses were observed therefore further development of CI-1040 was terminated due to poor antitumor activity. ${ }^{7}$

\section{PD-0325901}

A third MEK inhibitor, PD-0325901, has been developed and the results of the first clinical study were published in 2010. Though structurally similar to CI-1040, PD-0325901 is 50 times more potent at inhibiting MEK, has better solubility, and increased metabolic stability. ${ }^{10}$

The phase I trial of PD-0325901 enrolled 66 patients with advanced breast, colorectal, non-small cell lung cancer, and melanoma. Seven patients (10.6\%) developed transient blurred vision while taking PD-0325901. Of these seven, one patient reportedly developed optic neuropathy and two patients developed retinal vein occlusion after 4 months of therapy. The decision was made to amend the study protocol to add glaucoma, ocular hypertension, and any other significant ocular abnormality to the study's exclusion criteria. In addition, the dosing schedule was adjusted (5 days on/ 2 days off, instead of continuous dosing). Despite these changes, a third patient developed retinal vein occlusion after 9 months of therapy. At that time, further enrollment in the study was stopped due to retinal toxicity. ${ }^{10}$

In spite of the drug toxicity, antitumor activity appeared promising. Fifty-eight patients were evaluated for drug efficacy. Twenty-three patients had stable 
disease after two rounds of therapy and three melanoma patients had partial responses. ${ }^{10}$

\section{AZD6244}

The phase I clinical trial of the free-base suspension of AZD6244 was published in 2008 and enrolled 57 patients with numerous types of advanced cancer for which there was no proven curative or life-prolonging therapy. ${ }^{1,11}$ Transient and reversible blurred vision was reported in seven patients $(12 \%)$. Adjei et $a^{11}$ report that ophthalmologic examinations in these patients were unrevealing. Nine patients in this study achieved stable disease for $\geq 5$ months. One patient with malignant melanoma had $70 \%$ shrinkage of his tumors before developing brain metastasis. ${ }^{11}$

A phase I study was also conducted for an oral capsule formulation of AZD6244 to compare its bioavailability, efficacy, and safety to the initial formulation. About 10 of the $59(16.9 \%)$ patients enrolled reported ophthalmologic adverse events. Blurred vision was the most common symptom, though diplopia, dry eye, eyelid edema, increased lacrimation, visual disturbance, and scleral hemorrhage were also reported. The authors state that there were no patterns of abnormalities identified on ophthalmologic exams of these patients. ${ }^{12}$ Fifty-five patients were evaluated for antitumor activity of the drug. Ten patients (18.2\%) achieved stable disease for $\geq 16$ weeks. One patient, a 30 -year-old female with malignant melanoma, had a complete response. Her melanoma had progressed despite six cycles of chemotherapy prior to enrolling in this study. At the time of last evaluation, she had no recurrence of disease 2 years after completing 15 months of AZD6244. ${ }^{12}$

\section{GSK1120212}

GSK1120212, which goes by the generic name Trametinib and trade name Mekinist (GlaxoSmithKline, Research Triangle Park, NC, USA), is the first FDA-approved MEK inhibitor. On 29 May 2013, Trametinib was approved for the treatment of patients with unresectable or metastatic melanoma with BRAF V600E or V600K mutations. ${ }^{13}$

GSK1120212 was studied in a phase I multicenter clinical trial, which enrolled 206 patients with advanced solid tumors or lymphoma. Of note, exclusion criteria included history of retinal vein occlusion, central serous retinopathy, or glaucoma diagnosed within 1 month of starting the clinical trial. Ocular adverse events were reported in 31 patients $(15 \%)$, including 3 patients who developed central serous retinopathy and 1 patient who developed a retinal vein occlusion. All cases of central serous retinopathy were documented with OCT and resolved after the patients were taken off the drug.
None of the patients that developed ocular toxicity were restarted on the medication. Visual acuity in the patient with retinal vein occlusion improved with anti-VEGF injections. ${ }^{14}$ Reduction in tumor burden was seen in $10 \%$ of patients enrolled in the study. ${ }^{14,15}$

High response rates to GSK1120212 among patients with malignant melanoma, especially those with BRAF mutations, were demonstrated by this study and later confirmed in a phase II trial. Two cases of central serous retinopathy and no cases of retinal vein occlusion were reported in the phase II trial. ${ }^{16}$ A phase III trial randomized 322 patients with BRAF mutant melanoma to treatment with GSK1120212 vs standard chemotherapy. They found that the progression-free survival was 4.8 months in the MEK inhibitor group vs 1.5 months in the standard chemotherapy group. Nineteen (9\%) of the patients receiving GSK1120212 in this trial reported ocular adverse events, most being blurred vision. One patient was described as developing 'reversible chorioretinopathy.' There were no reports of retinal vein occlusion. ${ }^{17}$ Efficacy data from these clinical trials prompted the FDA approval of GSK1120212 for patients with BRAF mutant melanoma.

\section{RO5126766}

RO5126766 was the first in the class of dual RAF/MEK inhibitors to enter clinical trials. The phase I trial enrolled 52 patients with a large variety of advanced tumors. Ocular toxicities were reported in 26 patients (50\%), with 22 patients reporting blurred vision. Through the course of this study, the authors recognized an emerging pattern of blurred vision among patients enrolled in this as well as other MEK inhibitor trials and began to implement more thorough ophthalmologic evaluations. In doing so, they were able to identify 10 cases of serous retinal detachments on OCT imaging. This represents $38 \%$ of patients with ocular adverse events. The authors note that patients were not routinely evaluated with OCT from the beginning of the trial and therefore rates of serous retinal detachment are likely underestimated in their data. All of the ocular symptoms reversed spontaneously or with temporary interruption of systemic drug therapy. The authors recommend that comprehensive ophthalmologic assessments, including visual acuity, intraocular pressure measurement, fundoscopy, and OCT imaging, be implemented in future MEK inhibitor trials.

Of the 52 patients enrolled in this phase I trial, there were 3 partial responses, 16 patients with stable disease at 16 weeks, and 2 patients with stable disease for $>1$ year. All partial responses occurred in patients with malignant melanoma. ${ }^{18}$ 


\section{MEK 162}

MEK 162 is currently in phase II of clinical trials. As awareness of MEK inhibitor-associated retinopathy has spread among the oncology community, ophthalmic adverse events are receiving more attention. Recently in the Annals of Oncology, Urner-Block et al ${ }^{19}$ thoroughly evaluated 32 patients receiving treatment with MEK 162 for ophthalmologic complications. Every patient was sent to an ophthalmologist for a complete dilated eye exam including OCT imaging of the retina prior to starting treatment, 15 days into treatment and monthly from there on. Nineteen of the 32 (59\%) patients were found to have retinopathy described as multifocal grayish-yellow round lesions associated with serous retinal elevation. Only 8 of these $19(42 \%)$ patients were symptomatic. Those that were symptomatic described mild blurred vision. All patients had marked resolution of the lesions and blurred vision 2-6 weeks after starting treatment. This resolution occurred regardless of whether the patients continued the drug at the same dose or discontinued the drug. The authors concluded that there is a very high rate of MEK inhibitor-associated retinopathy even among asymptomatic patients. The retinopathy was generally mild, self-limited, and may not require discontinuation of treatment. ${ }^{19}$

\section{MEK inhibitors in the ophthalmology literature}

Despite high rates of ocular adverse events described in MEK inhibitor clinical trials, very few cases have been described in the ophthalmologic literature.

Velez-Montoya et $a l^{20}$ reported three cases of central serous retinopathy in patients enrolled in different MEK inhibitor clinical trials. Each of these cases resolved within days of stopping the drug without complications. ${ }^{20}$

A case report in 2013 described a 54-year-old female who developed multifocal central serous-like retinopathy after starting a MEK inhibitor for malignant melanoma. The patient presented for baseline eye exam prior to starting Dabrafenib (BRAF inhibitor) and Trametinib (MEK Inhibitor) and was found to have 20/20 vision OU with normal exam findings. She returned 3 weeks after starting the medications with visual acuity of 20/60 OD and 20/50 OS. Dilated fundus exam and OCT were suggestive of multifocal neurosensory retinal

detachments. Visual acuity improved and subretinal fluid resolved within 9 days of stopping both the medications. The MEK inhibitor was restarted 1 month later at a lower dose with recurrence of subretinal fluid and worsening visual acuity. These changes again resolved after stopping the MEK inhibitor for the second time. ${ }^{21}$

More recently, McCannel et $a^{22}$ published a case series of patients who developed neurosensory retinal detachments with MEK inhibitor use. All three of the patients described developed subfoveal neurosensory retinal detachments within 1 month of initiating drug therapy. Two of the three patients also developed anterior uveitis. All ocular adverse events were self-limited and all patients remained on the study drug, though dosing was lowered in one patient. ${ }^{22}$

\section{Mechanism of ocular toxicity}

As the ocular toxicity associated with MEK inhibitors is a relatively new discovery, very little is known about the mechanism of these ocular events. The majority of ocular toxicities reported with MEK inhibitors have occurred at the level of the retina with the two most common and sight-threatening events being retinal vein occlusion and subretinal fluid accumulation.

The occurrence of retinal vein occlusion, which resulted in termination of PD-0325901 clinical trials, prompted Huang et $a l^{23}$ to establish an animal model to investigate the mechanism of this adverse event. Intravitreal injections of PD-0325901 were administered to rabbits at various doses. At higher doses, all rabbits developed retinal vein occlusion within $24 \mathrm{~h}$. At 1 week post injection, the rabbits developed retinal edema, vascular attenuation, and retinal detachments. Oral PD-0325901 was administered to rats after which retinal gene expression analysis was conducted. No retinal occlusive events were seen in the rats; however, there were significant changes in retinal gene expression. An upregulation of genes involved in oxidative stress, blood retinal barrier breakdown, inflammatory response, as well as coagulation cascade activation were observed. The authors hypothesized that MEK inhibitors result in a combination of oxidative stress and a pro-thrombotic state, which together increase the risk for retinal vein occlusion. $^{23}$

Subretinal fluid accumulation in the absence of retinal vein occlusions has also been a common finding in patients treated with MEK inhibitors. RPE cells are responsible for maintaining the outer blood retinal barrier and preventing subretinal fluid accumulation. Jiang et al ${ }^{24}$ discovered the presence of aquaporin 1, a fluid transport channel, in RPE cells. They also demonstrated that the MEK/ERK pathway was involved in the regulation of aquaporin density within the RPE. ${ }^{24}$ The high incidence of serous retinal detachments described in patients taking MEK inhibitors suggests that MEK inhibition may alter the permeability of the RPE and thus disrupt its ability to serve as a barrier that prevents subretinal fluid accumulation. 


\section{Conclusions/Recommendations}

In this paper, we described two patients who developed retinal abnormalities during a course of treatment with MEK inhibitors. Our findings are similar to those published in the ophthalmologic literature. MEK inhibitors present a promising new option in the field of oncology but preliminary data suggest that these medications are associated with high rates of ocular toxicity (Table 1). More information is needed to fully characterize these toxicities and their pathogenesis. The development and utilization of these medications may result in an opportunity as well as challenge for ophthalmologists to diagnose and manage ocular complications in this patient population. Awareness of this class of medications and their ocular adverse effects among eye care professionals is the first step in managing ocular complications.

Numerous cases of subretinal fluid and RPE detachments associated with MEK inhibitors have been self-limited despite continuing systemic drug therapy. As in Case 1 and in previously reported cases, the RPE detachments were asymptomatic, not vision threatening, and resolved without local treatment or alteration of systemic drug therapy. In asymptomatic patients, it may be appropriate to continue the medication with more frequent follow-up to assess for worsening or improvement of retinal pathology. Should the retinal changes result in significant decrease in visual acuity without resolution over a period of 2-3 months, dose reduction or temporarily stopping the medication to allow resolution should be considered. Restarting the medication at a lower dose once the ocular adverse effects have resolved has been successful in preventing recurrence in many cases.

The patient in Case 2 developed CME, a complication that has not been previously reported in association with MEK inhibitors. Since this CME resolved completely with anti-inflammatory regimen of topical steroid and NSAID, this regimen may be considered as a management for retinal edema while continuing chemotherapy. Similar to the findings of McCannel et al, 22 in which two-thirds of patients had anterior uveitis, CME is likely inflammatory in etiology, justifying the use of topical or injectable antiinflammatory medications in these cases.

Retinal vein occlusion has been a rare but serious event associated with these medications that has the potential to result in permanent vision loss. In patients who have developed retinal vein occlusion, resulting visual acuity as well as the patient's response to treatment should be weighed. Consideration should be given to stopping the MEK inhibitor in these cases to prevent a second occlusive event from occurring in the same eye or in the fellow eye. The treatment of vein occlusions with anti-VEGF and
Table 1 The most common adverse events and ocular adverse events in MEK inhibitor clinical trials

\begin{tabular}{|c|c|c|}
\hline Clinical trial & $\begin{array}{l}\text { Most common } \\
\text { adverse events }\end{array}$ & $\begin{array}{l}\text { Frequency of ocular } \\
\text { adverse events }\end{array}$ \\
\hline CI-1040 Phase 1 & $\begin{array}{l}\text { Diarrhea }(33 \%) \\
\text { Asthenia }(23 \%) \\
\text { Rash }(14 \%) \\
\text { Nausea }(12 \%) \\
\text { Vomiting }(11 \%)\end{array}$ & None $(0 \%)$ \\
\hline CI-1040 Phase 2 & $\begin{array}{l}\text { Diarrhea }(57 \%) \\
\text { Nausea }(52 \%) \\
\text { Fatigue }(48 \%) \\
\text { Rash }(25 \%) \\
\text { Edema }(22 \%)\end{array}$ & 6 patients $(8.9 \%)$ \\
\hline PD-0325901 Phase 1 & $\begin{array}{l}\text { Rash }(79 \%) \\
\text { Fatigue }(61 \%) \\
\text { Diarrhea }(55 \%) \\
\text { Nausea }(38 \%) \\
\text { Edema }(26 \%)\end{array}$ & 7 patients $(10.6 \%)$ \\
\hline $\begin{array}{l}\text { AZD6244 Phase } 1 \\
\text { (oral suspension) }\end{array}$ & $\begin{array}{l}\text { Rash }(75 \%) \\
\text { Diarrhea }(61 \%) \\
\text { Nausea }(44 \%) \\
\text { Fatigue }(39 \%) \\
\text { Edema }(33 \%)\end{array}$ & 7 patients $(12.2 \%)$ \\
\hline $\begin{array}{l}\text { AZD Phase } 1 \\
\text { (oral capsule) }\end{array}$ & $\begin{array}{l}\text { Fatigue }(68 \%) \\
\text { Rash }(63 \%) \\
\text { Nausea }(54 \%) \\
\text { Diarrhea }(48 \%) \\
\text { Edema }(43 \%)\end{array}$ & 10 patients $(16.9 \%)$ \\
\hline GSK1120212 Phase 1 & $\begin{array}{l}\text { Rash }(83 \%) \\
\text { Diarrhea }(80 \%) \\
\text { Fatigue }(42 \%) \\
\text { Edema }(33 \%) \\
\text { Nausea }(29 \%)\end{array}$ & 31 patients $(15.0 \%)$ \\
\hline GSK1120212 Phase 2 & $\begin{array}{l}\text { Rash }(75 \%) \\
\text { Diarrhea }(52 \%) \\
\text { Nausea }(30 \%) \\
\text { Edema }(29 \%) \\
\text { Pruritis }(27 \%)\end{array}$ & 2 patients $(2.0 \%)$ \\
\hline GSK1120212 Phase 3 & $\begin{array}{l}\text { Rash }(57 \%) \\
\text { Diarrhea }(43 \%) \\
\text { Fatigue }(26 \%) \\
\text { Edema }(26 \%) \\
\text { Dermatitis }(19 \%)\end{array}$ & 19 patients $(9.0 \%)$ \\
\hline RO5126766 & $\begin{array}{l}\text { Rash }(94 \%) \\
\text { Elevated creatinine } \\
\text { phosphokinase } \\
\text { levels }(56 \%) \\
\text { Diarrhea }(52 \%) \\
\text { Blurred vision } \\
(42 \%) \\
\text { Edema }(31 \%)\end{array}$ & 22 patients $(42.3 \%)$ \\
\hline
\end{tabular}

steroid injections has proven useful in reducing macular edema and improving vision to varying degrees. These treatments can be used in cases in which vein occlusion and macular edema are believed to be an adverse events related to the MEK inhibitor. In addition, monitoring for 
systemic complications related to a suspected pro-thrombotic state is also advised.

In summary, we recommend that patients who will be started on a MEK inhibitor have a baseline retina examination with OCT and close follow-up in the first month of initiating the new drug as retinal changes may be asymptomatic. In patients being treated with these medications that present with ocular symptoms, dilated fundus exam should be completed to look for retinal abnormalities. OCT should be obtained to rule out pathology at the level of the RPE. FA should be utilized to monitor leakage and inflammation-related side effects. Patients on MEK inhibitors should be followed regularly to evaluate for ocular toxicity, especially in the first year of treatment. Management of retinal disease may vary depending on the suspected etiology and severity of symptoms. Treatment can range from close observation of asymptomatic pigment epithelial or neurosensory retinal detachments to topical anti-inflammatory drops for iritis or retinal edema, to intravitreal anti-VEGF or steroids for macular edema from retinal vein occlusion. Although patients may be reluctant to discontinue a potentially life-prolonging treatment as these patients have often failed to respond to all other chemotherapeutic agents, discontinuation or decreased dosing of medication should be considered for cases of severe sight-threatening ocular toxicity.

As utilization of MEK inhibitors increases in the field of oncology, more extensive ophthalmologic evaluation of these patients will be needed to better understand pathophysiology and long term prognosis for patients who develop ocular adverse events related to these medications.

\section{Conflict of interest}

The authors declare no conflict of interest.

\section{References}

1 Renouf DJ, Valzquez-Martin J, Simpson R, Siu L, Bedard P. Ocular toxicity of targeted therapies. J Clin Oncol 2012; 30: 3277-3284.

2 Zhao Y, Adjei AA. The clinical development of MEK Inhibitors. Nat Rev Clin Oncol 2014; 11: 385-400.

3 Cohen Y, Xing M, Mambo E, Guo Z, Wu G, Trink B et al. BRAF mutation in papillary thyroid carcinoma. J Natl Cancer Inst 2003; 95(8): 625-627.

4 Davies H, Bignell GR, Cox C, Stephens P, Edkins S, Clegg S et al. Mutations of the BRAF gene in human cancer. Nature 2002; 417(6892): 949-954.

5 Cheng Y, Zhang G, Li G. Targeting MAPK pathway in melanoma therapy. Cancer Metastasis Rev 2013; 32(3-4): 567-584

6 Akinleye A, Furqan M, Mukhi N, Ravella P, Delong L. MEK and the inhibitors: from bench to bedside. J Hematol Oncol 2013; 6: 27.
7 Wang D, Boerner SA, Winkler JD, LoRusso PM. Clincal experience of MEK inhibitors in cancer therapy. Biochim Biophys Acta 2007; 1773(8): 1248-1255.

8 Lorusso PM, Adjei AA, Varterasian M, Gadgeel S, Reid J, Mitchell DY et al. Phase I and pharmacodynamic study of the oral MEK inhibitor CI-1040 in patients with advanced malignancies. J Clin Oncol 2005; 23: 5281-5293.

9 Rinehart J, Adjei A, LoRusso P, Waterhouse D, Hecht R, Natale $\mathrm{R}$ et al. Multicenter phase II study of the oral MEK inhibitor, CI-1040, in patients with advanced non-small cell lung, breast, colon, and pan- creatic cancer. J Clin Oncol 2004; 22: $4456-4462$.

10 LoRusso PM, Krishnamurthi SS, Rinehart JJ, Nabell LN, Malburg L, Chapman P et al. Phase I pharmacokinetic and pharmacodynamic study of the oral MAPK/ERK kinase inhibitor PD-0325901 in patients with advanced cancers. Clin Cancer Res 2010; 16: 1924-1937.

11 Adjei AA, Cohen RB, Franklin W, Morris C, Wilson D, Molina JR et al. Phase I pharmacokinetic and pharmacodynamic study of the oral, small-molecule mitogen-activated protein kinase kinase $1 / 2$ inhibitor AZD6244 (ARRY-142886) in patients with advanced cancers. J Clin Oncol 2008; 26(13): 2139-2146.

12 Banerji U, Camidge DR, Verheul HMW, Agarwal R, Sarker $\mathrm{D}$, Kaye $\mathrm{S}$ et al. The first-in-human study of the hydrogen sulfate (Hyd-sulfate) capsule of the MEK1/2 inhibitor AZD6244 (ARRY-142886): a phase I open-label multicenter trial in patients with advanced cancer. Clin Cancer Res 2010; 16(5): 1613-1623

13 Trametinib-Food and Drug Administration. http://www. fda.gov/Drugs/InformationOnDrugs/ApprovedDrugs/ ucm354478.htm. Accessed 30 May 2013.

14 Infante JR, Fecher LA, Falchook GS, Nallapareddy S, Gordon MS, Becerra C et al. Safety, pharmacokinetic, pharmacodynamic, and efficacy data for the oral MEK inhibitor trametinib: a phase 1 dose-escalation trial. Lancet Oncol 2012; 13(8): 773-781.

15 Falchook GS, Lewis KD, Infante JR, Gordon MS, Vogelzang NJ, Demarini DJ et al. Activity of the oral MEK inhibitor trametinib in patients with advanced melanoma: a phase 1 dose-escalation trial. Lancet Oncol 2012; 13(8): 782-789.

16 Kim KB, Kefford R, Pavlick AC, Infante JR, Ribas A, Sosman JA et al. Phase II study of the MEK1/MEK2 inhibitor trametinib in patients with metastatic BRAF-mutant cutaneous melanoma previously treated with or without a BRAF inhibitor. J Clin Oncol 2013; 31(4): 482-489.

17 Flaherty KT, Robert C, Hersey P, Nathan P, Garbe C, Milhem $\mathrm{M}$ et al. Improved survival with MEK inhibition in BRAF-mutated melanoma. N Engl J Med 2012; 367(2): 107-114.

18 Martinez-Garcia M, Banerji U, Albanell J, Bahleda R, Dolly S, Kraeber-Bodere F et al. First-in-human, phase I doseescalation study of the safety, pharmacokinetics, and pharmacodynamics of RO5126766, a first-in-class dual MEK/RAF inhibitor in patients with solid tumors. Clin Cancer Res 2012; 18(17): 4806-4819.

19 Urner-Bloch U, Urner M, Stieger P, Galliker N, Winterton N, Zubel A et al. Transient MEK inhibitor-associated retinopathy in metastatic melanoma. Ann Oncol 2014; 25: 1437-1441.

20 Velez-Montoya R, Olsen J, Petrash M, Messersmith W, Mandava N, Oliver S. Acute onset central serous retinopathy with MEK inhibitor use for metastatic cancer. Invest Ophthalmol Vis Sci 2011; 52: E-Abstract 2153. 
21 Schoenberger SD, Kim SJ. Bilateral multifocal central serous-like chorioretinopathy due to MEK Inhibition for metastatic cutaneous melanoma. Case Rep Ophthalmol Med 2013; 2013: 673796.

22 McCannel TA, Chmielowski B, Finn RS, Goldman J, Ribas A, Wainberg ZA et al. Bilateral subfoveal neurosensory retinal detachment associated with MEK inhibitor use for metastatic cancer. JAMA Ophthalmol 2014; 132: 1005-1009.
23 Huang W, Yang AH, Matsumoto D, Collette W, Marroquin L, Ko M et al. PD0325901, a mitogen-activated protein kinase kinase inhibitor, produces ocular toxicity in rabbit animal model of retinal vein occlusion. J Ocul Pharmacol Ther 2009; 25: 519-530.

24 Jiang Q, Cao C, Lu S, Kivlin R, Wallin B, Chu W et al. MEK/ ERK pathway mediates UVB-induced AQP1 downregulation and water permeability impairment in human retinal pigment epithelial cells. Int J Mol Med 2009; 23: $771-777$. 